\title{
BASES OF THE QUANTUM CLUSTER ALGEBRA OF THE KRONECKER QUIVER
}

\author{
MING DING AND FAN XU
}

\begin{abstract}
We construct bar-invariant $\mathbb{Z}\left[q^{ \pm \frac{1}{2}}\right]$-bases of the quantum cluster algebra of the Kronecker quiver which are quantum analogues of the canonical basis, semicanonical basis and dual semicanonical basis of the cluster algebra of the Kronecker quiver in the sense of [14, 4, and [11] respectively. As a byproduct, we prove the positivity of the elements in these bases.
\end{abstract}

\section{INTRODUCTION}

Cluster algebras were introduced by S. Fomin and A. Zelevinsky [9] [10] in order to study total positivity in algebraic groups and the specialization of canonical bases of quantum groups at $q=1$. The study of $\mathbb{Z}$-bases of cluster algebras is important. There are many results involving the construction of $\mathbb{Z}$-bases of cluster algebras (for example, see [14] and [4] for cluster algebras of rank 2, [3] for finite type, [7] for type $\widetilde{A}$, [5] for $\widetilde{A}_{2}^{(1)}$, 6] for affine type and [11] for $Q$ without oriented cycles). As a quantum analog of cluster algebras, quantum cluster algebras were defined by $\mathrm{A}$. Berenstein and A. Zelevinsky in [1] in order to study canonical bases. A quantum cluster algebra is generated by a set of generators called cluster variables inside an ambient skew-field $\mathcal{F}$. Under the specialization $q=1$, the quantum cluster algebras are exactly cluster algebras.

Naturally, one may hope to construct $\mathbb{Z}\left[q^{ \pm \frac{1}{2}}\right]$-bases for quantum cluster algebras and further quantum analogues of bases of the corresponding cluster algebras. In this short note, we deal with the case of the quantum cluster algebra of the Kronecker quiver and construct various bar-invariant $\mathbb{Z}\left[q^{ \pm \frac{1}{2}}\right]$-bases by applying the $q$-deformation of the Caldero-Chapoton formula defined in [13] and the method in [14. Under the specialization $q=1$, these $\mathbb{Z}\left[q^{ \pm \frac{1}{2}}\right]$-bases are exactly the canonical basis, semicanonical basis and dual semicanonical basis of the corresponding cluster algebra in the sense of [14], 4] and [11] respectively. As a byproduct, we prove the positivity of the elements in these bases.

Recently, in [12, the author attached to certain element $w$ in Weyl group a subalgebra $U_{q}^{+}(w)$ of the positive part $U_{q}(n)$ of the universal enveloping algebra of a

2000 Mathematics Subject Classification. Primary 16G20, 20G42; Secondary 14M17.

Key words and phrases. quantum cluster algebra, $\mathbb{Z}\left[q^{ \pm \frac{1}{2}}\right]$-basis, positivity.

Fan Xu was partially supported by the Ph.D. Programs Foundation of Ministry of Education of China (No. 200800030058). 
Kac-Moody Lie algebra of type $\widetilde{A}_{1}^{(1)}$. The author proved that $U_{q}^{+}(w)$ is a quantum cluster algebra in the sense of Berenstein-Zelevinsky and gave explicit formulae for the cluster variables. Note that the cluster variables are some elements of $q$ deformation of dual canonical basis elements of $U_{q}^{+}(w)$. However it is not clear whether cluster monomials belong to the dual canonical basis. Thus comparing these $\mathbb{Z}\left[q^{ \pm \frac{1}{2}}\right]$-bases constructed in this note with the dual canonical basis of $U_{q}^{+}(w)$ becomes an interesting thing.

\section{Preliminaries}

2.1. Quantum cluster algebras. We begin with some of the terminology related to quantum cluster algebras. One can refer to [1] for more details. Let $L$ be a lattice of rank $m$ and $\Lambda: L \times L \rightarrow \mathbb{Z}$ a skew-symmetric bilinear form. We will need a formal variable $q$ and consider the ring of integer Laurent polynomials $\mathbb{Z}\left[q^{ \pm 1 / 2}\right]$. Define the based quantum torus associated to the pair $(L, \Lambda)$ to be the $\mathbb{Z}\left[q^{ \pm 1 / 2}\right]$-algebra $\mathcal{T}$ with a distinguished $\mathbb{Z}\left[q^{ \pm 1 / 2}\right]$-basis $\left\{X^{e}: e \in L\right\}$ and the multiplication given by

$$
X^{e} X^{f}=q^{\Lambda(e, f) / 2} X^{e+f} .
$$

It is easy to see that $\mathcal{T}$ is associative and the basis elements satisfy the following relations:

$$
X^{e} X^{f}=q^{\Lambda(e, f)} X^{f} X^{e}, X^{0}=1,\left(X^{e}\right)^{-1}=X^{-e} .
$$

It is known that $\mathcal{T}$ is an Ore domain, i.e., is contained in its skew-field of fractions $\mathcal{F}$. The quantum cluster algebra will be defined as a $\mathbb{Z}\left[q^{ \pm 1 / 2}\right]$-subalgebra of $\mathcal{F}$.

A toric frame in $\mathcal{F}$ is a map $M: \mathbb{Z}^{m} \rightarrow \mathcal{F} \backslash\{0\}$ of the form

$$
M(\mathbf{c})=\varphi\left(X^{\eta(\mathbf{c})}\right)
$$

where $\varphi$ is an automorphism of $\mathcal{F}$ and $\eta: \mathbb{Z}^{m} \rightarrow L$ is an isomorphism of lattices. By the definition, the elements $M(\mathbf{c})$ form a $\mathbb{Z}\left[q^{ \pm 1 / 2}\right]$-basis of the based quantum torus $\mathcal{T}_{M}:=\varphi(\mathcal{T})$ and satisfy the following relations:

$$
\begin{gathered}
M(\mathbf{c}) M(\mathbf{d})=q^{\Lambda_{M}(\mathbf{c}, \mathbf{d}) / 2} M(\mathbf{c}+\mathbf{d}), M(\mathbf{c}) M(\mathbf{d})=q^{\Lambda_{M}(\mathbf{c}, \mathbf{d})} M(\mathbf{d}) M(\mathbf{c}), \\
M(\mathbf{0})=1, M(\mathbf{c})^{-1}=M(-\mathbf{c}),
\end{gathered}
$$

where $\Lambda_{M}$ is the skew-symmetric bilinear form on $\mathbb{Z}^{m}$ obtained from the lattice isomorphism $\eta$. Let $\Lambda_{M}$ also denote the skew-symmetric $m \times m$ matrix defined by $\lambda_{i j}=\Lambda_{M}\left(e_{i}, e_{j}\right)$ where $\left\{e_{1}, \ldots, e_{m}\right\}$ is the standard basis of $\mathbb{Z}^{m}$. Given a toric frame $M$, let $X_{i}=M\left(e_{i}\right)$. Then we have

$$
\mathcal{T}_{M}=\mathbb{Q}\left(q^{1 / 2}\right)\left\langle X_{1}^{ \pm 1}, \ldots, X_{m}^{ \pm 1}: X_{i} X_{j}=q^{\lambda_{i j}} X_{j} X_{i}\right\rangle
$$

An easy computation shows that:

$$
M(\mathbf{c})=q^{\frac{1}{2} \sum_{i<j} c_{i} c_{j} \lambda_{j i}} X_{1}^{c_{1}} X_{2}^{c_{2}} \cdots X_{m}^{c_{m}}=: X^{(\mathbf{c})} \quad\left(\mathbf{c} \in \mathbb{Z}^{m}\right) .
$$

Let $\Lambda$ be an $m \times m$ skew-symmetric matrix and let $\tilde{B}$ be an $m \times n$ matrix, $n \leq m$. We call the pair $(\Lambda, \tilde{B})$ compatible if $\tilde{B}^{T} \Lambda=(D \mid 0)$ is an $n \times m$ matrix with $D=\operatorname{diag}\left(d_{1}, \cdots, d_{n}\right)$ where $d_{i} \in \mathbb{N}$ for $1 \leq i \leq n$. The pair $(M, \tilde{B})$ is called a 
quantum seed if the pair $\left(\Lambda_{M}, \tilde{B}\right)$ is compatible. Define the $m \times m$ matrix $E=\left(e_{i j}\right)$ by

$$
e_{i j}= \begin{cases}\delta_{i j} & \text { if } j \neq k \\ -1 & \text { if } i=j=k \\ \max \left(0,-b_{i k}\right) & \text { if } i \neq j=k\end{cases}
$$

For $n, k \in \mathbb{Z}, k \geq 0$, denote $\left[\begin{array}{l}n \\ k\end{array}\right]_{q}=\frac{\left(q^{n}-q^{-n}\right) \cdots\left(q^{n-r+1}-q^{-n+r-1}\right)}{\left(q^{r}-q^{-r}\right) \cdots\left(q-q^{-1}\right)}$. Let $\mathbf{c}=\left(c_{1}, \ldots, c_{m}\right) \in$ $\mathbb{Z}^{m}$ with $c_{k} \geq 0$. Define the toric frame $M^{\prime}: \mathbb{Z}^{m} \rightarrow \mathcal{F} \backslash\{0\}$ as follows:

$$
M^{\prime}(\mathbf{c})=\sum_{p=0}^{c_{k}}\left[\begin{array}{c}
c_{k} \\
p
\end{array}\right]_{q^{d_{k} / 2}} M\left(E \mathbf{c}+p \mathbf{b}^{k}\right), \quad M^{\prime}(-\mathbf{c})=M^{\prime}(\mathbf{c})^{-1} .
$$

where the vector $\mathbf{b}^{k} \in \mathbb{Z}^{m}$ is the $k$-th column of $\tilde{B}$. Then the quantum seed $\left(M^{\prime}, \tilde{B}^{\prime}\right)$ is defined to be the mutation of $(M, \tilde{B})$ in direction $k$. We say that two quantum seeds are mutation-equivalent if they can ba obtained from each other by a sequence of mutations. Let $\mathcal{C}=\left\{M^{\prime}\left(e_{i}\right): i \in[1, n]\right\}$ where $\left(M^{\prime}, \tilde{B}^{\prime}\right)$ is mutation-equivalent to $(M, \tilde{B})$. The elements of $\mathcal{C}$ are called cluster variables. Let $\mathcal{P}=\left\{M\left(e_{i}\right): i \in\right.$ $[n+1, m]\}$ and the elements of $\mathcal{P}$ are called coefficients. The quantum cluster algebra $\mathcal{A}_{q}\left(\Lambda_{M}, \tilde{B}\right)$ is the $\mathbb{Z}\left[q^{ \pm 1 / 2}\right]$-subalgebra of $\mathcal{F}$ generated by $\mathcal{C} \cup \mathcal{P}$. We associated with $(M, \tilde{B})$ the $\mathbb{Z}$-linear bar-involution on $\mathcal{T}_{M}$ by setting:

$$
\overline{q^{r / 2} M(\mathbf{c})}=q^{-r / 2} M(\mathbf{c}), \quad\left(r \in \mathbb{Z}, \mathbf{c} \in \mathbb{Z}^{n}\right) .
$$

It is easy to show that $\overline{X Y}=\bar{Y} \bar{X}$ for all $X, Y \in \mathcal{A}_{q}\left(\Lambda_{M}, \tilde{B}\right)$ and that each element of $\mathcal{C} \cup \mathcal{P}$ is bar-invariant.

2.2. The Kronecker quiver. Given a compatible pair $(\Lambda, \widetilde{B})$, we can associate a valued quiver (see [13, Section 2] for more details). Now we set $\Lambda=\left(\begin{array}{cc}0 & 1 \\ -1 & 0\end{array}\right)$ and $\widetilde{B}=\left(\begin{array}{cc}0 & 2 \\ -2 & 0\end{array}\right)$. The quiver $Q$ associated to this pair is the Kronecker quiver:

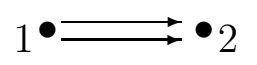

Let $k$ be a finite field with cardinality $|k|=q^{2}$. The category $\operatorname{rep}(k Q)$ of finitedimensional representations can be identified with the category of $\bmod -k Q$ of finitedimensional modules over the path algebra $k Q$. It is well-known (see [8]) that indecomposable $k Q$-module contains (up to isomorphism) three families: the indecomposable regular modules with dimension vector $\left(n d_{p}, n d_{p}\right)$ for $p \in \mathbb{P}_{k}^{1}$ of degree $d_{p}$ (in particular, denoted by $R_{p}(n)$ for $\left.d_{p}=1\right)$, the preprojective modules with dimension vector $(n-1, n)$ (denoted by $M(n))$ and the preinjective modules with dimension vector $(n, n-1)$ (denoted by $N(n))$. Here $n \in \mathbb{N}$. 
For $m \in \mathbb{Z} \backslash\{1,2\}$, set

$$
V(m)= \begin{cases}N(m-2) & \text { if } m \geq 3 \\ M(-m+1) & \text { if } m \leq 0\end{cases}
$$

Now, let $\mathcal{T}=\mathbb{Q}\left(q^{1 / 2}\right)\left\langle X_{1}^{ \pm 1}, X_{2}^{ \pm 1}: X_{1} X_{2}=q X_{2} X_{1}\right\rangle$ and $\mathcal{F}$ be the skew field of fractions of $\mathcal{T}$ and thus the quantum cluster algebra of the Kronecker quiver $\mathcal{A}_{q}(\Lambda, \tilde{B})$ (denoted by $\mathcal{A}_{q}(2,2)$ in the following) is the $\mathbb{Q}\left(q^{1 / 2}\right)$-subalgebra of $\mathcal{F}$ generated by the cluster variables $X_{k}, k \in \mathbb{Z}$, defined recursively by

$$
X_{m-1} X_{m+1}=q X_{m}^{2}+1 .
$$

The quantum Laurent phenomenon ([1]) implies that each $X_{k}$ belongs to the subring of $\mathcal{T}$ generated by $q^{ \pm 1 / 2}, X_{1}^{ \pm 1}, X_{2}^{ \pm 1}$. The explicit Laurent expansion of each $X_{k}$ in $X_{1}, X_{2}$ is given in [12] and [13].

Let $V$ be a representation of the Kronecker quiver with dimension vector $\underline{\operatorname{dim}} V=$ $\left(v_{1}, v_{2}\right)$. For $\mathbf{e}=\left(e_{1}, e_{2}\right) \in \mathbb{Z}_{\geq 0}^{2}$, denote by $G r_{\mathbf{e}}(V)$ the set of all subrepresentations $M$ of $V$ with $\underline{\operatorname{dim}} M=\mathbf{e}$. In [13], the author define the element $X_{V}$ of the quantum torus $\mathcal{T}$ by

$$
X_{V}=\sum_{\mathbf{e}} q^{-\frac{1}{2} d_{\mathbf{e}}^{V}}\left|G r_{\mathbf{e}}(V)\right| X^{\left(-v_{1}+2 v_{2}-2 e_{2}, 2 e_{1}-v_{2}\right)}
$$

where $d_{\mathbf{e}}^{V}=2 e_{1}\left(v_{1}-e_{1}\right)-2\left(2 e_{1}-e_{2}\right)\left(v_{2}-e_{2}\right)$. This formula is called a $q$-deformation of the Caldero-Chapoton formula ([2]). Here and the following, we simply write $X^{\mathbf{c}}$ instead of $X^{(\mathbf{c})}$ for $\mathbf{c} \in \mathbb{Z}^{2}$.

Theorem 2.1. [13] For any $m \in \mathbb{Z} \backslash\{1,2\}$, the $m$-th cluster variable $X_{m}$ of $\mathcal{A}_{q}(2,2)$ equals $X_{V(m)}$.

\section{Bases of the QuAntum Cluster algebra $\mathcal{A}_{q}(2,2)$}

In this section, we will construct various bar-invariant $\mathbb{Z}\left[q^{ \pm \frac{1}{2}}\right]$-bases of quantum cluster algebra $\mathcal{A}_{q}(2,2)$. Under the specialization $q=1$, these bases are just bases of the cluster algebra of the Kronecker quiver.

Definition 3.1. For any $\left(r_{1}, r_{2}\right)$ and $\left(s_{1}, s_{2}\right) \in \mathbb{Z}^{2}$, we write $\left(r_{1}, r_{2}\right) \preceq\left(s_{1}, s_{2}\right)$ if $r_{i} \leq s_{i}$ for $1 \leq i \leq 2$. Moreover, if there exists some $i$ such that $r_{i}<s_{i}$, then we write $\left(r_{1}, r_{2}\right) \prec\left(s_{1}, s_{2}\right)$.

Remark 3.2. By the definition of the $q$-deformation of the Caldero-Chapoton formula and the partial order in Definition 3.1. we obtain that the expansion of $X_{V(m)}$ have a minimal non-zero term $f\left(q^{\frac{1}{2}}, q^{-\frac{1}{2}}\right) X^{-\underline{\operatorname{dim}} V(m)}$ where $f\left(q^{\frac{1}{2}}, q^{-\frac{1}{2}}\right) \in \mathbb{Z}\left[q^{\frac{1}{2}}, q^{-\frac{1}{2}}\right]$. In fact, $f\left(q^{\frac{1}{2}}, q^{-\frac{1}{2}}\right)=1$ by the explicit expansion of $X_{V(m)}$ in [12] and [13, Proposition $1.1]$. 
Lemma 3.3. Let $R_{p}(1)$ be the indecomposable regular module of degree 1 as above. Then

$$
X_{R_{p}(1)}=X^{(-1,1)}+X^{(1,-1)}+X^{(-1,-1)} .
$$

Proof. Note that $R_{p}(1)$ contains the three submodules: $0, M(1)$ and $R_{p}(1)$. Thus the lemma immediately follows from the $q$-deformation of the Caldero-Chapoton formula.

By Lemma 3.3, the expression of $X_{R_{p}(1)}$ is independent of the choice of $p \in \mathbb{P}_{k}^{1}$ of degree 1 . Hence, we set

$$
X_{\delta}:=X_{R_{p}(1)}
$$

Definition 3.4. (1) The n-th Chebyshev polynomials of the first kind is the polynomial $F_{n}(x) \in \mathbb{Z}[x]$ defined by

$$
F_{0}(x)=1, F_{1}(x)=x, F_{2}(x)=x^{2}-2, F_{n+1}(x)=F_{n}(x) F_{1}(x)-F_{n-1}(x) \text { for } n \geq 2 \text {. }
$$

(2) The n-th Chebyshev polynomials of the second kind is the polynomial $S_{n}(x) \in$ $\mathbb{Z}[x]$ defined by

$$
S_{0}(x)=1, S_{1}(x)=x, S_{2}(x)=x^{2}-1, S_{n+1}(x)=S_{n}(x) S_{1}(x)-S_{n-1}(x) \text { for } n \geq 2 .
$$

It is obvious that $F_{n}(x)=S_{n}(x)-S_{n-2}(x)$. We denote $z=X_{\delta}, z_{n}=F_{n}(z), s_{n}=$ $S_{n}(z)$ for $n \geq 0$ and $z_{n}=s_{n}=0$ for $n<0$. Set

$$
\begin{aligned}
& \mathcal{B}=\left\{X_{m}^{a} X_{m+1}^{b} \mid m \in \mathbb{Z},(a, b) \in \mathbb{Z}_{\geq 0}^{2}\right\} \cup\left\{z_{n} \mid n \in \mathbb{N}\right\} \\
& \mathcal{S}=\left\{X_{m}^{a} X_{m+1}^{b} \mid m \in \mathbb{Z},(a, b) \in \mathbb{Z}_{\geq 0}^{2}\right\} \cup\left\{s_{n} \mid n \in \mathbb{N}\right\} \\
& \mathcal{D}=\left\{X_{m}^{a} X_{m+1}^{b} \mid m \in \mathbb{Z},(a, b) \in \mathbb{Z}_{\geq 0}^{2}\right\} \cup\left\{z^{n} \mid n \in \mathbb{N}\right\}
\end{aligned}
$$

Remark 3.5. (1) It is easy to check that $X^{(r, r)} X^{(s, s)}=X^{(r+s, r+s)}$ for any $r, s \in \mathbb{Z}$, thus the expansions of $z_{n}, s_{n}$ and $z^{n}$ have a minimal non-zero term $f\left(q^{\frac{1}{2}}, q^{-\frac{1}{2}}\right) X^{-(n, n)}$ according to the partial order in Definition 3.1.

(2) The elements $\mathbf{c}$ associated to these minimal non-zero terms $f\left(q^{\frac{1}{2}}, q^{-\frac{1}{2}}\right) X^{\mathbf{c}}$ in the expansion of the elements in the set $\mathcal{B}$ are different from each other. Indeed, it is easy to compute

$$
\mathbf{c}= \begin{cases}(b,-a) & \text { if } X^{\mathbf{c}}=X_{0}^{a} X_{1}^{b} ; \\ (a, b) & \text { if } X^{\mathbf{c}}=X_{1}^{a} X_{2}^{b} ; \\ (-b, a) & \text { if } X^{\mathbf{c}}=X_{2}^{a} X_{3}^{b} ; \\ a \cdot \underline{\operatorname{dim}} V(m)+b \cdot \underline{\operatorname{dim}} V(m+1) & \text { if } X^{\mathbf{c}}=X_{m}^{a} X_{m+1}^{b} \text { for } m \neq 0,1 \text { or } 2 ; \\ (n, n) & \text { if } X^{\mathbf{c}}=z_{n} .\end{cases}
$$

We note that there is at most one exceptional module in each dimension vector.

Now we define a ring homomorphism of the quantum cluster algebra $\mathcal{A}_{q}(2,2)$ :

$$
\sigma_{1}: \mathcal{A}_{q}(2,2) \longrightarrow \mathcal{A}_{q}(2,2)
$$


which sends $X_{m}$ to $X_{m+1}$ and $q^{ \pm \frac{1}{2}}$ to $q^{ \pm \frac{1}{2}}$. It is obviously an automorphism which preserves the defining relations. The following lemma is easy but important.

\section{Lemma 3.6.}

$$
\sigma_{1}\left(X_{\delta}\right)=X_{\delta}
$$

Proof. By Theorem 2.1 and the definition of the $q$-deformation of the CalderoChapoton formula, we have

$$
\begin{aligned}
X_{0}=X_{V(0)} & =X^{(2,-1)}+X^{(0,-1)}, \\
X_{3}=X_{V(3)} & =X^{(-1,2)}+X^{(-1,0)}, \\
X_{-1}=X_{V(-1)} & =X^{(3,-2)}+X^{(-1,-2)}+\left(q+q^{-1}\right) X^{(1,-2)}+X^{(-1,0)} .
\end{aligned}
$$

Following these identities and Lemma 3.3, one easily confirm the relations

$$
X_{\delta}=q^{\frac{1}{2}}\left(X_{0} X_{3}-q X_{1} X_{2}\right)=q^{\frac{1}{2}}\left(X_{-1} X_{2}-q X_{0} X_{1}\right) .
$$

Thus $\sigma_{1}\left(X_{\delta}\right)=\sigma_{1}\left(q^{\frac{1}{2}}\left(X_{-1} X_{2}-q X_{0} X_{1}\right)\right)=q^{\frac{1}{2}}\left(X_{0} X_{3}-q X_{1} X_{2}\right)=X_{\delta}$.

Lemma 3.7. For any $n \in \mathbb{Z}$,

$$
X_{n} X_{\delta}=q^{-\frac{1}{2}} X_{n-1}+q^{\frac{1}{2}} X_{n+1} .
$$

Proof. By an easy computation, we have:

$$
\begin{aligned}
X_{0} & =X^{(2,-1)}+X^{(0,-1)} \\
X_{-1} & =X^{(3,-2)}+X^{(-1,-2)}+\left(q+q^{-1}\right) X^{(1,-2)}+X^{(-1,0)} .
\end{aligned}
$$

Then by Lemma 3.3, it is easy to prove

$$
X_{0} X_{\delta}=q^{-\frac{1}{2}} X_{-1}+q^{\frac{1}{2}} X_{1} .
$$

Thus we can finish the proof by Lemma 3.6 and applying the automorphism $\sigma_{1}$.

Lemma 3.8.

$$
\overline{X_{\delta}}=X_{\delta}
$$

Proof. $\overline{X_{\delta}}=q^{-\frac{1}{2}}\left(\overline{X_{0} X_{3}}-q^{-1} \overline{X_{1} X_{2}}\right)=q^{-\frac{1}{2}}\left(X_{3} X_{0}-q^{-1} X_{2} X_{1}\right)=X_{\delta}$.

Remark 3.9. By Lemma 3.8 , we can verify that $\overline{z_{n}}=z_{n}, \overline{s_{n}}=s_{n}$ and $\sigma_{1}\left(z_{n}\right)=$ $z_{n}, \sigma_{1}\left(s_{n}\right)=s_{n}$.

The following proposition, which can be viewed as the quantum analogue of [14, Proposition 5.4], plays an essential role to construct $\mathbb{Z}\left[q^{ \pm \frac{1}{2}}\right]$-bases of the quantum cluster algebra $\mathcal{A}_{q}(2,2)$.

Proposition 3.10. (1) For $m>n \geq 1$ :

$$
\begin{aligned}
z_{n} z_{m} & =z_{m+n}+z_{m-n} \\
z_{n} z_{n} & =z_{2 n}+2 .
\end{aligned}
$$


(2) $m \geq 1$ and $n \in \mathbb{Z}$ :

$$
X_{n} z_{m}=q^{\frac{m}{2}} X_{n+m}+q^{-\frac{m}{2}} X_{n-m} .
$$

(3) For $m \geq 0$ and $n \in \mathbb{Z}$ :

$$
\begin{aligned}
X_{n} X_{n+2 m} & =q^{m} X_{n+m}^{2}+\sum_{l=0}^{m-1} q^{-m+2 l+1} \sum_{k=l+1}^{m} z_{2(m-k)}, \\
X_{n} X_{n+2 m+1} & =q^{m} X_{n+m} X_{n+m+1}+\sum_{l=0}^{m-1} q^{-m+2 l+\frac{1}{2}} \sum_{k=l+1}^{m} z_{2(m-k)+1} .
\end{aligned}
$$

Proof. The proof of (1) follows from the inductive relations in the definition of Chebyshev polynomials.

As for (2), we make induction on $m$. If $m=1$, the equation in (2) is a direct corollary of Lemma 3.7. We assume that (2) holds for $m \leq k$. For $m=k+1$, we have

$$
\begin{aligned}
X_{n} z_{k+1}= & X_{n}\left(z_{k} z_{1}-z_{k-1}\right) \\
= & \left(q^{\frac{k}{2}} X_{n+k}+q^{-\frac{k}{2}} X_{n-k}\right) z_{1}-\left(q^{\frac{k-1}{2}} X_{n+k-1}+q^{-\frac{k-1}{2}} X_{n-k+1}\right) \\
= & q^{\frac{k}{2}} X_{n+k} z_{1}+q^{-\frac{k}{2}} X_{n-k} z_{1}-\left(q^{\frac{k-1}{2}} X_{n+k-1}+q^{-\frac{k-1}{2}} X_{n-k+1}\right) \\
= & q^{\frac{k}{2}}\left(q^{\frac{1}{2}} X_{n+k+1}+q^{-\frac{1}{2}} X_{n+k-1}\right)+q^{-\frac{k}{2}}\left(q^{\frac{1}{2}} X_{n-k+1}+q^{-\frac{1}{2}} X_{n-k-1}\right) \\
& -\left(q^{\frac{k-1}{2}} X_{n+k-1}+q^{-\frac{k-1}{2}} X_{n-k+1}\right) \\
= & q^{\frac{k+1}{2}} X_{n+k+1}+q^{-\frac{k+1}{2}} X_{n-k-1} .
\end{aligned}
$$

This proves (2). Now we prove (3). If $m=0$, it is obvious. If $m=1$, by the recurrence relations (2.2), we have

$$
X_{n} X_{n+2}=q X_{n+1}^{2}+1
$$

We have proved that the equation $X_{0} X_{3}=q X_{1} X_{2}+q^{-\frac{1}{2}} z$ (see (3.1)) holds, thus by Lemma 3.6, we have

$$
X_{n} X_{n+3}=q X_{n+1} X_{n+2}+q^{-\frac{1}{2}} z .
$$

Now we assume that equations in (3) hold for $m \leq k$. For $m=k+1$, by Lemma 3.7, we have

$$
X_{n} X_{n+2 k+2}=q^{-\frac{1}{2}} X_{n}\left(X_{n+2 k+1} z_{1}-q^{-\frac{1}{2}} X_{n+2 k}\right) .
$$

Following the inductive assumption, it is equal to

$$
q^{-\frac{1}{2}}\left(q^{k} X_{n+k} X_{n+k+1}+\sum_{l=0}^{k-1} q^{-k+2 l+\frac{1}{2}} \sum_{i=l+1}^{k} z_{2(k-i)+1}\right) z_{1}-q^{-1}\left(q^{k} X_{n+k}^{2}+\sum_{l=0}^{k-1} q^{-k+2 l+1} \sum_{i=l+1}^{k} z_{2(k-i)}\right) .
$$

Using Lemma 3.7 again and (1) of this proposition, it is

$$
q^{k-\frac{1}{2}} X_{n+k}\left(q^{\frac{1}{2}} X_{n+k+2}+q^{-\frac{1}{2}} X_{n+k}\right)+\sum_{l=0}^{k-1} q^{-k+2 l} \sum_{i=l+1}^{k} z_{2(k-i)+1} z_{1}
$$




$$
\begin{aligned}
& -q^{-1}\left(q^{k} X_{n+k}^{2}+\sum_{l=0}^{k-1} q^{-k+2 l+1} \sum_{i=l+1}^{k} z_{2(k-i)}\right) \\
= & q^{k+1} X_{n+k+1}^{2}+\sum_{l=0}^{k} q^{-k+2 l} \sum_{i=l+1}^{k+1} z_{2(k-i+1)} .
\end{aligned}
$$

Similarly, by Lemma 3.7, we have

$$
X_{n} X_{n+2 k+3}=q^{-\frac{1}{2}} X_{n}\left(X_{n+2 k+2} z_{1}-q^{-\frac{1}{2}} X_{n+2 k+1}\right)
$$

Using the equation $\left(^{*}\right)$ and similar proof, we obtain

$$
X_{n} X_{n+2 k+3}=q^{k+1} X_{n+k+1} X_{n+k+2}+\sum_{l=0}^{k} q^{-k+2 l-\frac{1}{2}} \sum_{i=l+1}^{k+1} z_{2(k-i+1)+1} .
$$

Remark 3.11. By Lemma 3.8 and properties of bar-invariant, we can easily obtain the similar results for $z_{m} X_{n}, X_{n+2 m} X_{n}, X_{n+2 m} X_{n}$.

We similarly define the quantized version of the definition of positivity in [14].

Definition 3.12. A nonzero element $x \in \mathcal{A}_{q}(2,2)$ is positive if for every $m \in \mathbb{Z}$, all the coefficients in the expansion of $x$ as a Laurent polynomial in $\left\{x_{m}, x_{m+1}\right\}$ belong to $\mathbb{N}\left[q^{ \pm \frac{1}{2}}\right]$.

Corollary 3.13. Every element in $\mathcal{B}, \mathcal{S}$ and $\mathcal{D}$ is a positive element of quantum cluster algebra $\mathcal{A}_{q}(2,2)$.

Proof. By Lemma 3.3 and the fact $z_{n}(x)=s_{n}(x)-s_{n-2}(x)$, we only need to prove every element in $\mathcal{B}$ is positive. By the definition of $\sigma_{1}$ and Remark 3.9, it is enough to prove the positivity in $\left\{x_{1}, x_{2}\right\}$. We prove it by induction. For convenience, we write down the following equations according to Proposition 3.10 and Remark 3.11; For $m \geq 1$ :

$$
X_{1} z_{m}=q^{\frac{m}{2}} X_{1+m}+q^{-\frac{m}{2}} X_{1-m}
$$

For $m \geq 0$ :

$$
\begin{aligned}
(3.3) X_{1} X_{1+2 m}= & q^{m} X_{1+m}^{2}+q^{-m+1} \sum_{k=1}^{m} z_{2(m-k)}+q^{-m+3} \sum_{k=2}^{m} z_{2(m-k)} \\
& +\cdots+q^{m-3} \sum_{k=m-1}^{m} z_{2(m-k)}+q^{m-1} ; \\
(3.4) X_{1} X_{2+2 m}= & q^{m} X_{1+m} X_{2+m}+q^{-m+\frac{1}{2}} \sum_{k=1}^{m} z_{2(m-k)+1}+q^{-m+\frac{5}{2}} \sum_{k=2}^{m} z_{2(m-k)+1} \\
& +\cdots+q^{m-\frac{7}{2}} \sum_{k=m-1}^{m} z_{2(m-k)+1}+q^{m-\frac{3}{2}} z_{1} .
\end{aligned}
$$


$(3.5)$

$$
\begin{aligned}
X_{1} X_{1-2 m}= & q^{-m} X_{1-m}^{2}+q^{m-1} \sum_{k=1}^{m} z_{2(m-k)}+q^{m-3} \sum_{k=2}^{m} z_{2(m-k)} \\
& +\cdots+q^{-m+3} \sum_{k=m-1}^{m} z_{2(m-k)}+q^{-m+1} \\
X_{1} X_{-2 m}= & q^{-m-1} X_{-m} X_{1-m}+q^{m-\frac{1}{2}} \sum_{k=1}^{m} z_{2(m-k)+1}+q^{m-\frac{5}{2}} \sum_{k=2}^{m} z_{2(m-k)+1} \\
& +\cdots+q^{-m+\frac{7}{2}} \sum_{k=m-1}^{m} z_{2(m-k)+1}+q^{-m+\frac{3}{2}} z_{1} .
\end{aligned}
$$

It is easy to check that

$$
\left\{X_{-2}, X_{-1}, X_{0}, X_{1}, X_{2}, z_{1}, z_{2}\right\}
$$

are positive elements in $\left\{x_{1}, x_{2}\right\}$. Now assume that

$$
\left\{X_{-2 m}, X_{-2 m+1}, \cdots, X_{2 m-1}, X_{2 m}, z_{1}, \cdots, z_{2 m}\right\}
$$

are positive elements in $\left\{x_{1}, x_{2}\right\}$. Then by (3.2), (3.3) and (3.4), we know that $X_{2 m+1}, X_{2 m+2}$ and $X_{-1-2 m}$ are positive. Thus we obtain that $z_{2 m+1}$ is positive by (3.1). Therefore by (3.5), we have that $X_{-2 m-2}$ is positive. Again by (3.2), we know that $X_{2 m+3}$ is positive. Thus we get $z_{2 m+2}$ is positive by (3.1) again. Throughout the above discussions we obtain

$$
\left\{X_{-2 m-2}, X_{-2 m-1}, \cdots, X_{2 m+1}, X_{2 m+2}, z_{1}, \cdots, z_{2 m+2}\right\}
$$

are positive elements in $\left\{x_{1}, x_{2}\right\}$. The proof is finished.

Remark 3.14. In fact, by [15] [12] [13, the positivity in the cluster variables is obvious, then applying the equation $X_{1} z_{m}=q^{\frac{m}{2}} X_{1+m}+q^{-\frac{m}{2}} X_{1-m}$, we can deduce the positivity in the elements $z_{m}$ for any $m \in \mathbb{N}$. Here, we give an alternative proof without needing the explicit expansions of cluster variables.

Theorem 3.15. The sets $\mathcal{B}, \mathcal{S}$ and $\mathcal{D}$ are $\mathbb{Z}\left[q^{ \pm \frac{1}{2}}\right]$-bases of the quantum cluster algebra $\mathcal{A}_{q}(2,2)$.

Proof. Note that if $\mathcal{B}$ is a $\mathbb{Z}\left[q^{ \pm \frac{1}{2}}\right]$-basis of the quantum cluster algebra $\mathcal{A}_{q}(2,2)$, then $\mathcal{S}$ and $\mathcal{D}$ are naturally $\mathbb{Z}\left[q^{ \pm \frac{1}{2}}\right]$-bases of quantum cluster algebra $\mathcal{A}_{q}(2,2)$ because there are have unipotent matrix transformations between $\left\{z_{n} \mid n \in \mathbb{N}\right\},\left\{s_{n} \mid n \in \mathbb{N}\right\}$ and $\left\{z^{n} \mid n \in \mathbb{N}\right\}$. In the following, we will focus on the set $\mathcal{B}$ and prove it is a $\mathbb{Z}\left[q^{ \pm \frac{1}{2}}\right]$-basis of the quantum cluster algebra $\mathcal{A}_{q}(2,2)$.

By Proposition 3.10, we obtain that any element of the quantum cluster algebra $\mathcal{A}_{q}(2,2)$ can be a $\mathbb{Z}\left[q^{ \pm \frac{1}{2}}\right]$-combination of the elemnets in the set $\mathcal{B}$. Thus we only need to prove the elemnets in $\mathcal{B}$ are $\mathbb{Z}\left[q^{ \pm \frac{1}{2}}\right]$-independent. 
By Remark 3.5, we know that the elements c associated to these minimal non-zero terms $f\left(q^{\frac{1}{2}}, q^{-\frac{1}{2}}\right) X^{\mathbf{c}}$ in the expansion of the elements in the set $\mathcal{B}$ are different from each other. Now we suppose that a finite $\mathbb{Z}\left[q^{ \pm \frac{1}{2}}\right]$-combination of the elemnets in the set $\mathcal{B}$ is equal to 0 . Let $S \subset \mathbb{Z}^{2}$ be the set of all $\alpha$ such that the corresponding element occurs with a non-zero coefficient in this $\mathbb{Z}\left[q^{ \pm \frac{1}{2}}\right]$-combination. If $S$ is non-empty, pick a minimal element $\alpha \in S$, by Remark 3.2 and Remark 3.5 , we know that $X^{\alpha}$ does not occur in the expansion of any other element in above equation which gives a contradiction. This completes the proof of the theorem.

Set

$$
\begin{aligned}
\mathcal{B}^{\prime} & =\left\{q^{-\frac{a b}{2}} X_{m}^{a} X_{m+1}^{b} \mid m \in \mathbb{Z},(a, b) \in \mathbb{Z}_{\geq 0}^{2}\right\} \cup\left\{z_{n} \mid n \in \mathbb{N}\right\} \\
\mathcal{S}^{\prime} & =\left\{q^{-\frac{a b}{2}} X_{m}^{a} X_{m+1}^{b} \mid m \in \mathbb{Z},(a, b) \in \mathbb{Z}_{\geq 0}^{2}\right\} \cup\left\{s_{n} \mid n \in \mathbb{N}\right\} \\
\mathcal{D}^{\prime} & =\left\{q^{-\frac{a b}{2}} X_{m}^{a} X_{m+1}^{b} \mid m \in \mathbb{Z},(a, b) \in \mathbb{Z}_{\geq 0}^{2}\right\} \cup\left\{z^{n} \mid n \in \mathbb{N}\right\} .
\end{aligned}
$$

Then we can obtain the following corollary.

Corollary 3.16. The sets $\mathcal{B}^{\prime}, \mathcal{S}^{\prime}$ and $\mathcal{D}^{\prime}$ are bar-invariant $\mathbb{Z}\left[q^{ \pm \frac{1}{2}}\right]$-bases of the quantum cluster algebra $\mathcal{A}_{q}(2,2)$.

\section{An REPRESEntation-THEORETiC INTERPRETATion of THE ELEMENT $s_{n}$}

Recall we denote by $R_{p}(n)$ the indecomposable regular modules with dimension vector $(n, n)$ for $n \geq 1$ and some $p \in \mathbb{P}_{k}^{1}$ of degree 1 . In this section, we will prove that $s_{n}$ is equal to $X_{n \delta}$ for every $n \in \mathbb{N}$. The following proposition shows the Laurent expansion of $X_{m}$ in $A_{q}(2,2)$.

Proposition 4.1. (12, [13]) For every $n \geq 0$, we have

$$
\begin{aligned}
& X_{-n}=X^{(n+2,-n-1)}+\sum_{p+r \leq n}\left[\begin{array}{c}
n-r \\
p
\end{array}\right]_{q}\left[\begin{array}{c}
n+1-p \\
r
\end{array}\right]_{q} X^{(2 r-n, 2 p-n-1)} \\
& X_{n+3}=X^{(-n-1, n+2)}+\sum_{p+r \leq n}\left[\begin{array}{c}
n-r \\
p
\end{array}\right]_{q}\left[\begin{array}{c}
n+1-p \\
r
\end{array}\right]_{q} X^{(2 p-n-1,2 r-n)} .
\end{aligned}
$$

Lemma 4.2. For every $n \in \mathbb{N}$, we have

$$
s_{n}=q^{\frac{n}{2}} X_{1} X_{n+3}-q^{\frac{n}{2}+1} X_{2} X_{n+2} .
$$

Proof. It is easy to check that $s_{1}=q^{\frac{1}{2}} X_{1} X_{4}-q^{\frac{3}{2}} X_{2} X_{3}$. Assume that it holds for $n \leq k$, then

$$
\begin{aligned}
s_{k+1} & =s_{k} s_{1}-s_{k-1} \\
& =\left(q^{\frac{k}{2}} X_{1} X_{k+3}-q^{\frac{k}{2}+1} X_{2} X_{k+2}\right) s_{1}-\left(q^{\frac{k-1}{2}} X_{1} X_{k+2}-q^{\frac{k+1}{2}} X_{2} X_{k+1}\right) \\
& =q^{\frac{k}{2}} X_{1}\left(q^{\frac{1}{2}} X_{k+4}+q^{-\frac{1}{2}} X_{k+2}\right)-q^{\frac{k}{2}+1} X_{2}\left(q^{\frac{1}{2}} X_{k+3}+q^{-\frac{1}{2}} X_{k+1}\right) \\
& -\left(q^{\frac{k-1}{2}} X_{1} X_{k+2}-q^{\frac{k+1}{2}} X_{2} X_{k+1}\right) \\
& =q^{\frac{k+1}{2}} X_{1} X_{k+4}-q^{\frac{k+1}{2}+1} X_{2} X_{k+3} .
\end{aligned}
$$


Denote the quantum binomial coefficients $\left(\begin{array}{l}n \\ r\end{array}\right)_{q}=\frac{\left(q^{n}-1\right)\left(q^{n-1}-1\right) \cdots\left(q^{n-r+1}-1\right)}{\left(q^{r}-1\right) \cdots(q-1)}$ and take $\left(\begin{array}{l}n \\ 0\end{array}\right)_{q}=1$ for any $n \in \mathbb{Z},\left(\begin{array}{l}n \\ l\end{array}\right)_{q}=0$ for any $l<0$, and $\left(\begin{array}{l}n \\ l\end{array}\right)_{q}=0$ for any $0 \leq n<l$. Then we have the following theorem proved in [15]:

Theorem 4.3. [15, Theorem 4.6] Let $\boldsymbol{e}=(a, b)$ for $(a, b) \in \mathbb{Z}_{\geq 0}^{2}$. Then for $n \geq 1$,

$$
\left|G r_{e}\left(R_{p}(n)\right)\right|=\left(\begin{array}{l}
n-a \\
n-b
\end{array}\right)_{q^{2}}\left(\begin{array}{l}
b \\
a
\end{array}\right)_{q^{2}} .
$$

Proposition 4.4. $X_{R_{p}(n)}=s_{n}$ for $n \in \mathbb{N}$.

Proof. By the $q$-deformation of the Caldero-Chapoton formula and Theorem 4.3, we have

$$
\begin{aligned}
X_{R_{p}(n)} & =\sum_{(a, b)} q^{-\frac{1}{2} d_{(\mathbf{a}, \mathbf{b})}^{R_{p}(n)}}\left|G r_{(a, b)}\left(R_{p}(n)\right)\right| X^{(n-2 b, 2 a-n)} \\
& =\sum_{(a, b)} q^{(a-b)(n-b)}\left(\begin{array}{c}
n-a \\
n-b
\end{array}\right)_{q^{2}} q^{(a-b) a}\left(\begin{array}{l}
b \\
a
\end{array}\right)_{q^{2}} X^{(n-2 b, 2 a-n)} \\
& =\sum_{(a, b)}\left[\begin{array}{c}
n-a \\
n-b
\end{array}\right]_{q}\left[\begin{array}{l}
b \\
a
\end{array}\right]_{q}^{(n-2 b, 2 a-n)} \\
& =\sum_{p+r \leq n}\left[\begin{array}{c}
n-r \\
p
\end{array}\right]_{q}\left[\begin{array}{c}
n-p \\
r
\end{array}\right]_{q} X^{(2 p-n, 2 r-n)} .
\end{aligned}
$$

On the other hand, by Proposition 4.1, we have

$$
\begin{aligned}
q^{\frac{n}{2}} X_{1} X_{n+3} & =q^{\frac{n}{2}} X_{1} X^{(-n-1, n+2)}+q^{\frac{n}{2}} X_{1} \sum_{p+r \leq n}\left[\begin{array}{c}
n-r \\
p
\end{array}\right]_{q}\left[\begin{array}{c}
n+1-p \\
r
\end{array}\right]_{q} X^{(2 p-n-1,2 r-n)} \\
& =q^{n+1} X^{(-n, n+2)}+\sum_{p+r \leq n}\left[\begin{array}{c}
n-r \\
p
\end{array}\right]_{q}\left[\begin{array}{c}
n+1-p \\
r
\end{array}\right]_{q} q^{r} X^{(2 p-n, 2 r-n)} .
\end{aligned}
$$

And

$$
\begin{aligned}
q^{\frac{n}{2}+1} X_{2} X_{n+2} & =q^{\frac{n}{2}+1} X_{2} X^{(-n, n+1)}+q^{\frac{n}{2}+1} X_{2} \sum_{p+r \leq n-1}\left[\begin{array}{c}
n-1-r \\
p
\end{array}\right]_{q}\left[\begin{array}{c}
n-p \\
r
\end{array}\right]_{q} X^{(2 p-n, 2 r+1-n)} \\
& =q^{n+1} X^{(-n, n+2)}+\sum_{p+r \leq n-1}\left[\begin{array}{c}
n-1-r \\
p
\end{array}\right]_{q}\left[\begin{array}{c}
n-p \\
r
\end{array}\right]_{q} q^{n-p+1} X^{(2 p-n, 2 r+2-n)} \\
& =q^{n+1} X^{(-n, n+2)}+\sum_{p+r \leq n}\left[\begin{array}{c}
n-r \\
p
\end{array}\right]_{q}\left[\begin{array}{c}
n-p \\
r-1
\end{array}\right]_{q} q^{n-p+1} X^{(2 p-n, 2 r-n)} .
\end{aligned}
$$

Note that it is easy to confirm the following identity:

$$
\left[\begin{array}{c}
n+1-p \\
r
\end{array}\right]_{q} q^{r}=\left[\begin{array}{c}
n-p \\
r
\end{array}\right]_{q}+\left[\begin{array}{c}
n-p \\
r-1
\end{array}\right]_{q} q^{n-p+1} .
$$


Then by Lemma 4.2, we have

$$
\begin{aligned}
s_{n} & =q^{\frac{n}{2}} X_{1} X_{n+3}-q^{\frac{n}{2}+1} X_{2} X_{n+2} \\
& =\sum_{p+r \leq n}\left[\begin{array}{c}
n-r \\
p
\end{array}\right]_{q}\left[\begin{array}{c}
n-p \\
r
\end{array}\right]_{q} X^{(2 p-n, 2 r-n)} \\
& =X_{R_{p}(n)} .
\end{aligned}
$$

\section{ACKNOWLEDGEMENTS}

The authors would like to thank Professor Jie Xiao for many helpful discussions.

\section{REFERENCES}

[1] A. Berenstein, A. Zelevinsky, Quantum cluster algebras, Advances in Mathematics, vol. 195 (2005), 405-455.

[2] P. Caldero, F. Chapoton, Cluster algebras as Hall algebras of quiver representations, Comment. Math. Helv. 81 (2006), no. 3, 595-616.

[3] P. Caldero and B. Keller, From triangulated categories to cluster algebras, Invent. math. 172 (2008), no. 1, 169-211.

[4] P. Caldero, A. Zelevinsky, Laurent expansions in cluster algebras via quiver representations, Mosc. Math. J. 6 (2006), no. 3, 411-429.

[5] G. Cerulli Irelli, Canonically positive basis of cluster algebras of type $\widetilde{A}_{2}^{(1)}$, arXiv:0904.2543.

[6] M. Ding, J. Xiao and F. Xu, Integral bases of cluster algebras and representations of tame quivers, arXiv:0901.1937.

[7] G. Dupont, Generic variables in acyclic cluster algebras, arXiv: 0811.2909.

[8] V. Dlab, C. Ringel, Indecomposable Representations of Graphs and Algebras, Mem. Amer. Math. Soc., 173 (1976).

[9] S. Fomin and A. Zelevinsky, Cluster algebras. I. Foundations, J. Amer. Math. Soc. 15 (2002), no. 2, 497-529.

[10] S. Fomin and A. Zelevinsky, Cluster algebras. II. Finite type classification, Invent. Math. 154 (2003), no. 1, 63-121.

[11] C. Geiss, B. Leclerc and J. Schröer, Generic bases for cluster algebras and the Chamber Ansatz, arXiv: 1004.2781 .

[12] P. Lampe, A quantum cluster algebra of Kronecker type and the dual canonical basis, arXiv:1002.2762,

[13] Dylan Rupel, On quantum Analogue of the Caldero-Chapoton Formula, arXiv:1003.2652.

[14] P. Sherman, A. Zelevinsky, Positivity and canonical bases in rank 2 cluster algebras of finite and affine types, Moscow Math. J., 4 (2004), no. 4, 947-974.

[15] C. Szanto, On the cardinalities of Kronecker quiver grassmainnians, arXiv:0903.1928.

Institute for advanced study, Tsinghua University, Beijing 100084, P. R. China

E-mail address: m-ding04@mails.tsinghua.edu.cn (M.Ding)

Department of Mathematical Sciences, Tsinghua University, Beijing 100084, P. R. China

E-mail address: fanxu@mail.tsinghua.edu.cn (F.Xu) 